\title{
Impact of Timing of Lung resection on Survival for Clinical Stage I and II Lung Cancer
}

\section{Background}

Lung cancer is the second most common cancer in both men and women, and comprise $13 \%$ of all new cancers. It is by far the leading cause of cancer death among men and women. Each year, more people die of lung cancer than of colon, breast, and
prostate cancers combined, $(1,2)$ There is an increasing effort towards early detection of prostate cancers combined. $(1,2)$ There is an increasing effort towards early detection of
lung cancer, since it is a curable cancer if diagnosis and treatment are performed in a timely manner.(3)

Surgical resection is the most effective treatment for lung cancer in early stages, between diagnosis and resection of early-stage lung cancer do not exist.

\section{Objectives}

This work aims to assess whether increasing time between diagnosis/first doctor visit and surgery for early stage non-sma poorer survival.

\section{Methods}

We identified a retrospective cohort of incident lung cancer cases who had surgical and no prior radiation or chemotherapy. We assessed overall survival (OS) and predictors included a) time from first contact to surgery; and b) time from diagnosis to surgery. The associlur between dale of diagnosis and date of first contact of a health assessed using multivariable Cox proportional hazard. We investigated four cutof points: surgery within 15 days, 30 days, 60 days and 90 days. We controlled for sociodemographic characteristics as well as clinical outcomes.

Results

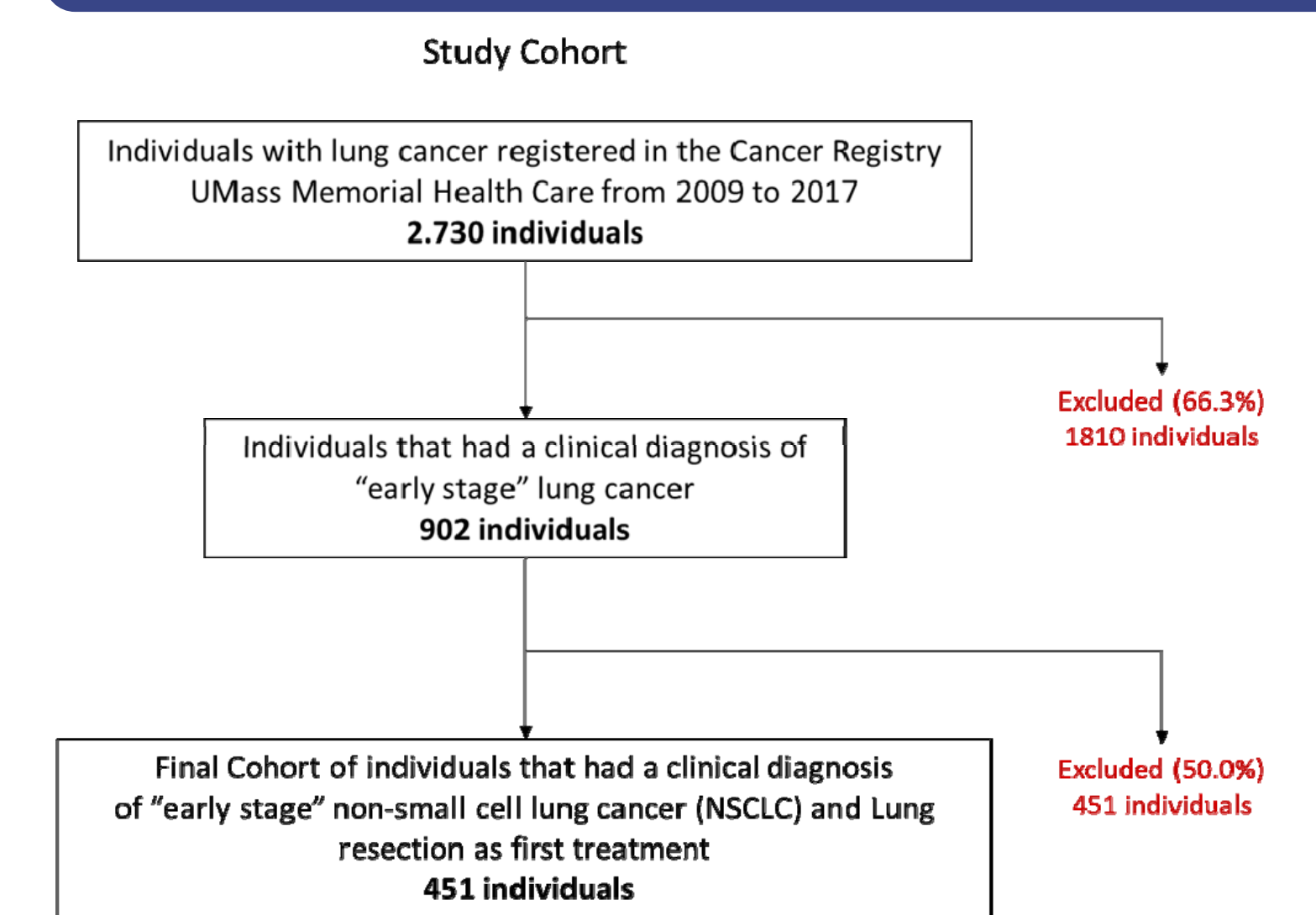

Figure 1. Diagram showing schema of study

Our cohort comprised 451 patients. The age average was 67 years, $61 \%$ female $94.7 \%$ white, and $9 \%$ never smoked Clinical Slage $1 A$ and $1 B$ corresponded stage it was $76 \%$.

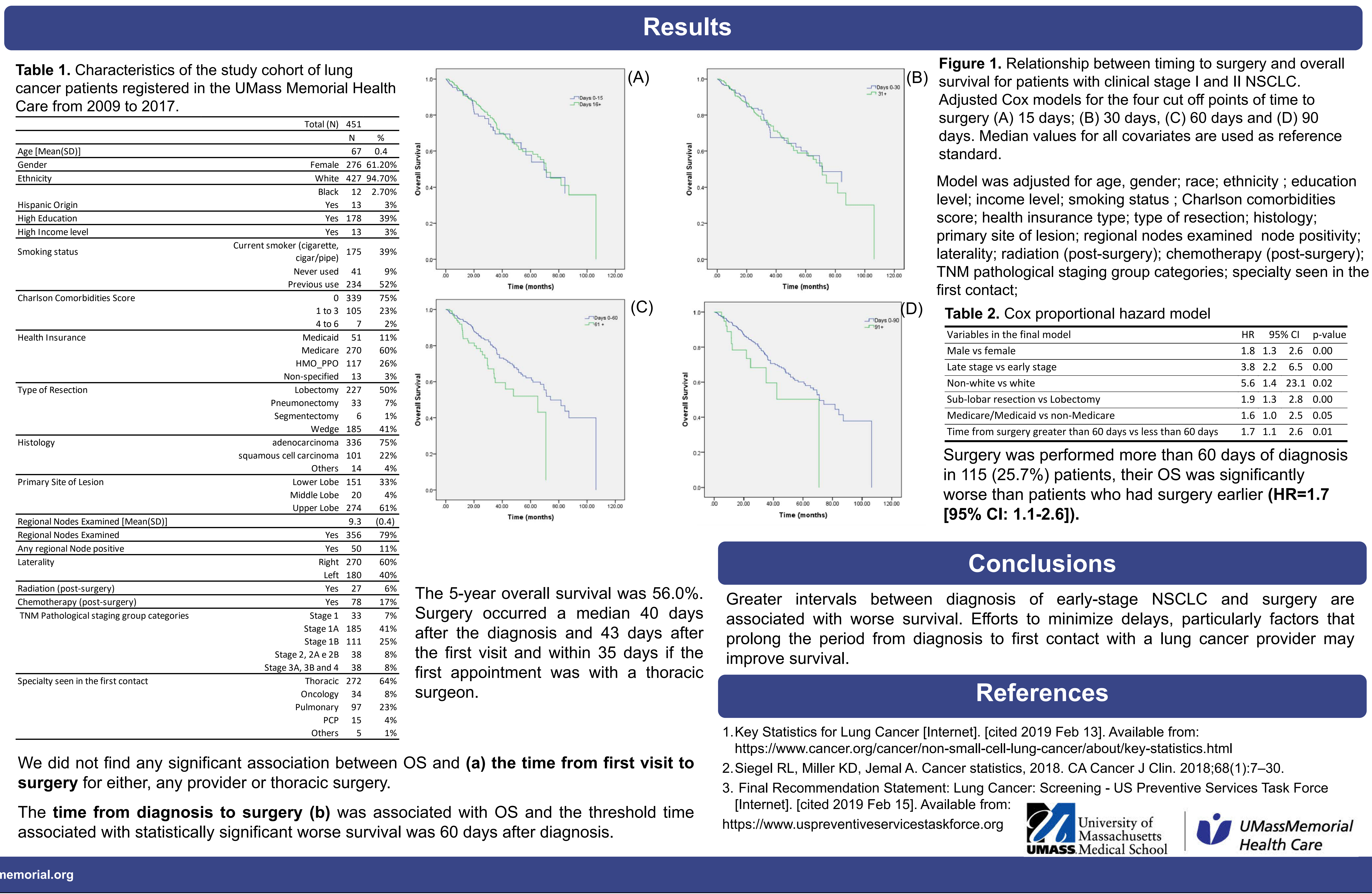

